\title{
The European Cross-Border Health Data Exchange: Focus on Clinically Relevant Data
}

\author{
Sari PALOJOKI ${ }^{\mathrm{a}, 1}$, Anne VAKKURI ${ }^{\mathrm{b}}$ and Riikka VUOKKO ${ }^{\mathrm{a}}$ \\ ${ }^{\text {a }}$ The Ministry of Social Affairs and Health, Finland \\ ${ }^{b}$ Helsinki University Hospital (HUS), Peijas Hospital, Finland
}

\begin{abstract}
The eHealth Digital Service Infrastructure (eHDSI) is an infrastructure ensuring the continuity of care for European citizens while they are travelling abroad in the EU. We present the Finnish readiness of implementing datasets of diagnosis, vaccinations and medication summary in a case study, and discuss challenges emerging from the national perspective. International harmonized standards are a key element in the smooth development of European information exchange.
\end{abstract}

Keywords. Cross-border Health Data Exchange, Medication, Vaccination, Diagnosis, Classification

\section{Introduction}

In Europe, the right of a patient for cross-border healthcare is governed in Directive 2011/24/EU of the European Parliament and of the Council of 9 March 2011 [1]. Long lasting efforts have been devoted to establish a European Union (EU) wide, common infrastructure for cross-border health data exchange to support healthcare services [2]. Currently, efficient data sharing and usage is among the major priorities in the EU [3], and it is a subject, which is more actual than ever due to the ongoing COVID-19 pandemic [4].

The eHealth Digital Service Infrastructure (eHDSI) is the initial deployment and operation of services for cross-border health data exchange under the Connecting Europe Facility (CEF). eHDSI deploys the core and generic services for Patient Summary (PS) and ePrescription as the first European application area. These generic services include the implementation of data exchange at country level, and the respective core services at EU level. Together, these enable provision of Cross Border eHealth Information Services (CBeHIS). [2,5] The European Commission invested in the cross-border health data exchange with the epSOS project during 2008-2014, and with several other initiatives following its proposed model. In practice, efficient health data exchange has encountered challenges, and at the time of writing, seven countries have joined eHDSI [5-7]. The major barriers have been identified in Member States, and are as follows e.g., [2]: lack of standard EHR system, different implementation of EU regulations, and different information workflows among national infrastructure and healthcare organizations.

1 Corresponding author, Dr. Sari Palojoki, P.O. Box 33, 00023 Helsinki, Finland, sari.palojoki@gmail.com. 
The European PS guideline [8] specifies a dataset of essential information for unplanned or emergency care with the aim to improve patient safety and quality of care. Though there are more datasets in the PS, we have chosen to focus on some of the most clinically relevant datasets to review herein. We present the Finnish readiness of implementing datasets of medical problems, vaccinations and medication summary in a case study, and discuss challenges emerging from the national perspective. At the time of writing, urgent needs in health data exchange focus on vaccination data. Effective vaccines are needed in order to restrain the pandemic. [9] Moreover, medical problems and medication summary are among the essential contents of patient data [10]. Thus, our research questions are (1) What is the level of readiness for Finnish development of summary datasets in medical problems, vaccinations and medication in the European PS Guidelines, and (2) Which classifications are used in Finland to structure these datasets?

\section{Methods}

In this article, we research EU PS data sets and their readiness in terms of implementation with a case study in the Finland. Case studies are designed to suit the case and research question, and publications demonstrate wide diversity in study design. Case study provides methodological flexibility through the incorporation of different paradigmatic positions, study designs, and methods but it requires careful planning and reporting. [11] We produce a descriptive case analysis based on our research questions [12]. To form our research data, we collected documentation of development work and specifications available for our document analysis. We build up our case study by using the specifications of EU commission on the eHDSI [8]. A comparison of EU specifications to Finnish data structures were analysed based on the national data specifications available in the National Code Service (NCS) [13-15].

In order to build our case study, we provide contextual information as proposed by Kaplan and Duchon [12] to support reliability of the observations. In Finland, all patient data is stored in national Kanta information services. In Kanta requirements, crucial patient data is structured for nationally uniform use. The national data structures and classifications are published and maintained in the NCS, which is a crucial cornerstone for building a successful digital health infrastructure in Finland. [13] Our research team consists of subject-matter experts in the research topic: two of the authors act as informatics and data structure experts, who were developing the NCS and the common data structures and are currently steering national development efforts at the ministerial context. The third author is a senior medical officer and a clinician having first-hand experience on the clinical data sets in Finland.

Finland joined eHDSI in 2019 by implementing e-prescription service. Finland is preparing to introduce the PS in a few years [5]. According to the PS Guidelines Release 2 , the PS is a "data set of essential and understandable health information" that is made available "to deliver safe patient care during unscheduled care and planned care with its maximal impact in the unscheduled care". The PS consists of two types of information. Basic information, which is the minimum information needed from the clinical point of view, and extended information that is desirable from the clinical point of view. 


\section{Results}

Our results describe vaccination, medical problems and medication data elements in the EU PS (see Table 1). Vaccination data is described in the conceptual grouping "History of Past Illnesses". The data set contains information concerning each disease against which immunization was given, the brand name of the vaccination given to the patient, unique identifier of the vaccination and the date when the immunization was received. Table 1 illustrates that the vaccination data elements in the current version of the PS are identified by extended data set principles answering for cross-border information needs in clinical setting. In comparison to present Finnish common data elements for vaccination, equivalence is apparent. These data elements are already implemented in Finland. At the time of writing, EU requirements on the classifications are not yet available. In Finland, classifications in use for vaccination data are of national origin, and thus, require further development to harmonize with EU requirements.

List of current medical problems consists of three basic data elements as described in the Table 1. Problems/diagnosis that fit under these conditions are conditions that may have a chronic or relapsing course, conditions for which the patient receives repeat medications and conditions that are persistent and serious contraindications for classes of medication. Problem ID is a unique identifier of the medical problem. Onset time is the date when the patient first experienced the condition or symptoms of the disease. When comparing data elements with the situation in Finland, it can be concluded that all respective data structures have been in use for years. There are two international classifications, WHO International Classifications for Diseases (ICD-10) and International Classification of Primary Care (ICPC2). ICD-10 serves more widely for the purpose of medical coding. Current developments in the NCS include preparations for the introduction of the next version of WHO ICD (ICD-11).

Table 1. Vaccinations, medical problems and medication summary data elements in the EU PS, and respective data elements and classifications in use in Finland.

\begin{tabular}{|c|c|c|}
\hline EU Patient Summary Data Sets & $\begin{array}{l}\text { Implementation status } \\
\text { in Finland }\end{array}$ & Classifications in Finland \\
\hline \multicolumn{3}{|l|}{ Vaccination data elements } \\
\hline Vaccination & $\mathrm{X}$ & Disease vaccine protects against \\
\hline $\begin{array}{l}\text { The brand name of the vaccination given } \\
\text { to the patient }\end{array}$ & $\mathrm{X}$ & Vaccine products \\
\hline Vaccinations ID & $\mathrm{X}$ & \\
\hline Vaccination Date & $\mathrm{X}$ & \\
\hline \multicolumn{3}{|l|}{ List of current medical problems } \\
\hline Problem/diagnosis description & $\mathrm{X}$ & ICD-10, ICPC-2 (code name) \\
\hline Problem ID (code) & $\mathrm{X}$ & ICD-10, ICPC-2 \\
\hline Onset time & $\mathrm{X}$ & \\
\hline \multicolumn{3}{|l|}{ Medication summary } \\
\hline Active ingredient & $\mathrm{X}$ & $\mathrm{ATC}^{1}$ (code name) \\
\hline Active ingredient ID (code) & $\mathrm{X}$ & ATC \\
\hline Strength of the medicinal product & $\mathrm{X}$ & \\
\hline Pharmaceutical dose form & $\mathrm{X}$ & Dose form $\left(\mathrm{EDQM}^{2}\right)$ \\
\hline Number of units per intake & $\mathrm{X}$ & \\
\hline Frequency of intakes & $\mathrm{X}$ & \\
\hline Duration of treatment & $(\mathrm{X})$ & \\
\hline Date of onset of treatment & $\mathrm{X}$ & \\
\hline
\end{tabular}

\footnotetext{
${ }^{1}$ WHO ATC $=$ Anatomical Therapeutic Chemical Classification System; ${ }^{2} \mathrm{EDQM}=$ European Directorate for the Quality of Medicines
} 
Medication summary (see Table 1) consists of several data elements. Active ingredient is a substance that alone or in combination with other ingredients produces the intended activity of a medicinal product. The "Active Ingredient" element is usually filled in if the "Active ingredient ID code" is available, but it may be provided as free text as well. Strength of the medicinal product is the content of the active ingredient which is expressed quantifiable per dosage unit, per unit of volume or per unit of weight. Pharmaceutical dose form is the form in which a pharmaceutical product is presented in the product package. The number of units stands for each intake that the patient is taking. Frequency of the intakes defines intakes patient should take. The duration of treatment is constituted of three optional subfields: start date, end date and length of treatment. End date and length of treatment are mutually exclusive. Date of onset of treatment is date when the patient needs to start taking the prescribed medicine. When assessing the preparation situation in Finland with a view to the introduction of the PS, the readiness for medication summary is good. Only the duration of treatment is documented with other type of data elements (start of treatment element and optional end of treatment data element). However, there are also challenges with medication data related to the long history of classification development in Finland. Many of the medication-related classifications have been developed nationally, which makes mapping necessary for the EU implementation. Moreover, some of the existing classifications in the NCS will be replaced in the future by the new options. The standards developed by the International Organization for Standardization (ISO) for the identification of medicinal products (IDMP) will be implemented, which will provide a more accurate classification option. This concerns, for example, the WHO Anatomical Therapeutic Chemical Classification System (ATC) that is likely to become replaced when new standard is implemented. [1314]

\section{Discussion}

Our case study explored Finnish readiness for implementing the PS as expediently aligned with the eHDSI initiative of the PS services across European countries. Overall, the Finnish readiness for the PS is well prepared. In future, this European joint effort plays an increasing role in providing safer and better cross-border healthcare.

When Finland's situation in development of data elements is assessed, it is possible to identify certain challenges that the pioneer position of digitalization has brought with it. Finland started developing the national system relatively early [13], which in some cases meant that national classifications had to be drawn up. International standards were localized and applied where possible, but in many cases local development work was introduced. [13-14] The past eHealth development in Finland pose challenges in relation to semantic interoperability requirements that can be predicted in the EU development for the coming years. Currently, in Finland, Snomed Clinical Terminology (CT) has been assessed and it is localized in some clinical domains for the purpose of providing uniform data structures. However, in well-established clinical domains, with a long history of data structuration, data harmonization can be increased by semantic mapping. The disadvantage is that reconciling terminologies requires data content analysis and concept mapping that is costly and time-consuming [13-15]

As we move towards an international exchange of information [5-6], solutions will have to be found to remedy these inconsistencies at different levels of interoperability. Today, at European level, it is essential to continue successful semantic cooperation to 
define common medical terminology standards. A future issue that needs to be considered will be what kind of role ICD-11 and Snomed CT will play in structuring medical information. It is evident that a lot of multidisciplinary cooperation and impact assessment is required.

Concerning recommendations for future research, our case study suggests that further exploration of the PS is essential. Taken a relative slow implementation of PS more user experiences and descriptions are needed. The European Guideline on crossborder exchange of the PS and dataset has been taken as the basis for projects in Europe. The dataset is currently in its third iteration but only few countries have implemented it. $[2,5-6,8]$ We propose a more in-depth study of the possibilities of international classifications and terminologies, for example, to facilitate data compatibility in the future. Our conclusion is that the experiences related to the joining of eHDSI need to be analyzed more than at present in order to address the factors hindering the implementation of eServices. International harmonized standards are a key element in the smooth development of common European information exchange.

\section{References}

[1] Directive 2011/24/EU of the European Parliament and of the Council of 9 March 2011 on the application of patients' rights in cross-border healthcare. Available at: https:/eur-lex.europa.eu/eli/dir/2011/24/oj.

[2] Nalin M, Baroni I, Faiella G, Romano M, Matrisciano F, Gelenbe E, Martinez DM, Dumortier J, Natsiavas P, Votis K, Koutkias V, Tzovaras D, Clemente F. The European cross-border health data exchange roadmap: Case study in the Italian setting. J Biomed Inform. 2019 Jun;94:103183.

[3] European Commission. Commission priorities for 2019-24. Available at: https://ec.europa.eu/info/strategy/priorities-2019-2024_en.

[4] The Lancet Digital Health. Transparency during global health emergencies. Lancet Digit Health. 2020 Sep;2(9):e441.

[5] European Commission. eHealth DSI Operations. Available at: https://ec.europa.eu/cefdigital/wiki/display/EHOPERATIONS/eHealth+DSI+Operations+Home

[6] European Commission. eHealth: Digital health and care. Available at: https://ec.europa.eu/health/ehealth/electronic_crossborder healthservices en.

[7] Moharra M, Almazán C, Decool M, Nilsson AL, Allegretti N , Seven M. Implementation of a cross-border health service: physician and pharmacists' opinions from the epSOS project. Fam Pract. 2015 Oct;32(5):564-7. doi: 10.1093/fampra/cmv052. Epub 2015 Jul 6. PMID: 26148726.

[8] European Commission. Create the eHDSI Patient Summary content. The Patient Summary Guidelines (Release 2). Available at: https://ec.europa.eu/cefdigital/wiki/display/EHOPERATIONS/05.01.

[9] ECDC: COVID-19 vaccination and 5rioritization strategies in the EU/EEA. 2020. Available at: https://www.ecdc.europa.eu/sites/default/files/documents/Overview-of-EU_EEA-UK-vaccinationdeployment-plans.pdf.

[10] Adane K, Gizachew M, Kendie S. The role of medical data in efficient patient care delivery: a review. Risk Manag Healthc Policy. 2019 Apr 24;12:67-73. doi: 10.2147/RMHP.S179259. PMID: 31114410.

[11] Hyett N, Kenny A, Dickson-Swift V. Methodology or method? A critical review of qualitative case study reports. Int J Qual Stud Health Well-being. 2014 May 7;9:23606. doi: 10.3402/qhw.v9.23606. PMID: 24809980 ; PMCID: PMC4014658.

[12] Kaplan B., Duchon D. Combining Qualitative and Quantitative Methods in Information Systems Research: A Case Study. MIS Quarterly. 1988; 12(4), 571-586. doi:10.2307/249133.

[13] Vuokko R, Mäkelä-Bengs P, Härkönen M. Improving health care service delivery with a national code service. Stud Health Technol Inform. 2014;205:323-7. PMID: 25160199.

[14] Mäkelä-Bengs P, Vuokko R. Code service in social and health care - process description of the THL code service. THL Directions 2013, 19. http://urn.fi/URN:ISBN:978-952-302-042-9.

[15] European Union. Assessing SNOMED CT for Large Scale eHealth Deployments in the EU. 2016. Available at: https://assess-ct.eu/fileadmin/assess_ct/final_brochure/assessct_final_brochure.pdf. 